\title{
Mapping Mental Models into Interfaces of Interactive Systems
}

\author{
Abdulrazaq Alsuhail Almutairi \\ Information \& Computer Center \\ The Public Authority for Applied Education and Training \\ The Ministry of Education \\ State of Kuwait
}

\begin{abstract}
The computer plays an important role in our lives in every aspect. It is used in communication, academic research, business activities, and many other areas. It is required in any of those areas where we, as humans, interact with machines. This interaction is normally achieved through a Graphical User Interface (GUI). GUI represents a mode of communication between human and machines, allowing users to tell machines specific operations to be performed and feedback the state of the machine. There are some challenges in building a GUI that can be easily understood by users so they can use it without errors or mistakes. Mental models represent a very important concept in the humancomputer interaction area. Considering mental models when building a GUI plays an important role in providing effective solutions to end users. This paper presents an approach that facilitates the consideration of mental models, when building a solution that requires an interaction between users and computers.
\end{abstract}

\section{Introduction}

Humans have sophisticated sensory systems such as touch, sight and other senses used to perceive the world around them. Despite the existence of these sensory systems, human perceptions evolve and are enriched by experience. Gained experience helps in interpreting different situations, even if there is lack of a full understanding of a specific situation. Proposing a solution that does not take into consideration how users associate their prior experiences to use it will impact its effectiveness. In the area of cognitive science, the association of prior experience is known as mental models. Immersion [1] explained the mental model in their white paper as follows:

"The mental model we have for a particular experience guides our response to new situations and compensates for information or context that the current situation may not provide. If the mental model is incomplete or inaccurate, our response to a new situation can be misguided, faulty, or inadequate."

At the point when software designers build new products or applications, they are communicating thoughts that they think will guide users to associate their prior experience. The thought is known as a "conceptual model" and when appropriately implemented in the application design, it will coordinate a mental model that end users position and employ. Effective software applications have the ability to map this bond effectively, such applications normally achieve end-user satisfaction. In other words, software designers need to create interfaces and interaction methods that facilitate creating a more accurate mental model for the system to be interacted with. Kurtz [2] described the importance of mental models in building interactive systems as follows:

"Having an inaccurate model of how a system works may cause problems while interacting with the system. By studying how people create mental models of interactive systems and by designing interactive systems that help the user create a more accurate mental model of the system, usability will improve."

The idea of mental models is built based on mental representations of things around us. Those models may be employed in training users how to interact with systems. In this paper, an approach is proposed, to map the mental models users possess to system interfaces they will interact with. The methodology considers some of the ten usability heuristics for user interface design to achieve that mapping.

The second section in this paper presents a brief background. The third section goes through related work carried out in other research. The fourth section provides an explanation for the proposed methodology and how to apply it. The fifth and sixth sections provide examples of applying the methodology in building part of the interfaces in interactive systems. The final section presents the conclusion and suggested future work.

\section{Background}

\subsection{Mental Model}

The theory of mental models was introduced by Craik in the early 1940's [3]. The theory states that the mind builds a small-scale model of what is around them to anticipate and understand events around them. Since introduction of that theory, cognitive scientists have argued that the human mind builds the mental models based on understanding, imagination, knowledge, and the perception of discourse. They studied how to 
develop computer systems that provide a model of one domain presenting an analogy for another domain.

Johnson-Laird [4] proposed a theory based on Craik's assumption, stating that humans must possess a model of a certain phenomenon to perceive it. This means that mental models do not necessarily represent a visual representation of an event or experience. Khella [5] described the relation between mental models and phenomenon as follow:

"The only constraint for a mental model is that it has a similar structure to the phenomenon it represents. An ideal mental model explains all the aspects of the phenomenon the individual is interacting with. A referentially isomorphic mapping exists between the mental model and the real one."

The mental models hypothesis knocked down the old psychological speculations and presumptions that people utilize some sort of prepositional rationale to make inductions about the world. Johnson-Laird [4] suggests that people construct mental models of a situation and then make an assumption and come to a conclusion about which model is the best fit. Individuals have a tendency to take care of issues by utilizing earlier data and learning about comparable issues, if comparability exists between the structure of the two models.

As an example of how humans construct mental models, let us consider a typical android phone user asked to try using an apple phone. The user at first will try to find a back button to navigate back to previous pages of an application and will get frustrated and stuck for a while. The fact that the user has been using android phones builds a mental model for the back option in almost all programs, and associates this model with the back action. Absence of such a button will make the user struggle in navigating to previous pages.

\subsection{Usability Heuristics For User Interface Design}

In the early 1990's, Nielsen and Molish [6] proposed ten principles (heuristics) which can be considered as a guide, when developing a user interface. These principles can also be used as an evaluation method that helps in identifying usability issues in a user interface. The heuristics are as follow:

Visibility of system status: The user interface should always report to users within a reasonable time about what is happening, through a suitable feedback.
Match between system and the real world: The system should communicate with users using the same language they speak. In other words, the system should use expressions and terminologies familiar to the user, rather than technical terms.

User control and freedom: User interfaces need to provide a way of recovery, if they action something by mistake. The system should give users the freedom to stop a process they selected by mistake and restore the system to its previous state.

Consistency and standards: Different views in a user interface should be consistent in terms of style, terminologies, components and should follow platform conventions.

Error prevention: It is preferred to consider having an error prevention mechanism rather than displaying an appropriate error message. For example, it is better to present a confirmation dialogue before deleting a record.

Recognition rather than recall: Users do not need to remember all the steps and options required to perform certain functionality. They should be able to recognize actions and options required to perform desired functionalities once they interact with the system. In other words, an effective user interface reduces the user's memory load by clearly showing objects, actions, options and instructions.

Flexibility and efficiency of use: The system should be tailored to suit both normal and expert users. It should have the capabilities to allow customizations that facilitate and accelerate frequent actions and operations.

Aesthetic and minimalist design: Dialogues need to be simple and short and not contain irrelevant information.

Help users recognize, diagnose, and recover from errors: Errors should be reported in plain language that clearly explains the problem, and suggests a solution.

Help and documentation: Users should be able to easily find help and documentation related to the task they want to carry out. The contents of help should be in the form of simple steps rather than a lot of text.

\section{Mental Model Mapping Approach}

\subsection{Overview}

The proposed approach in this paper can be considered as a guide to building a conceptual model that maps a user's mental model into a designer's model. Fig.1 shows different models involved in the proposed methodology. The designer's model is the model developed inside the system, this may go through 
different sources of information such as profiles, configurations, error reporting, and logs history. The second model is the user's mental model that exists before building the system; the mental model is built through experience obtained by using similar systems, and the user's domain knowledge. The conceptual model can be considered as an exact and reliable representation of the objective framework held by the designer.

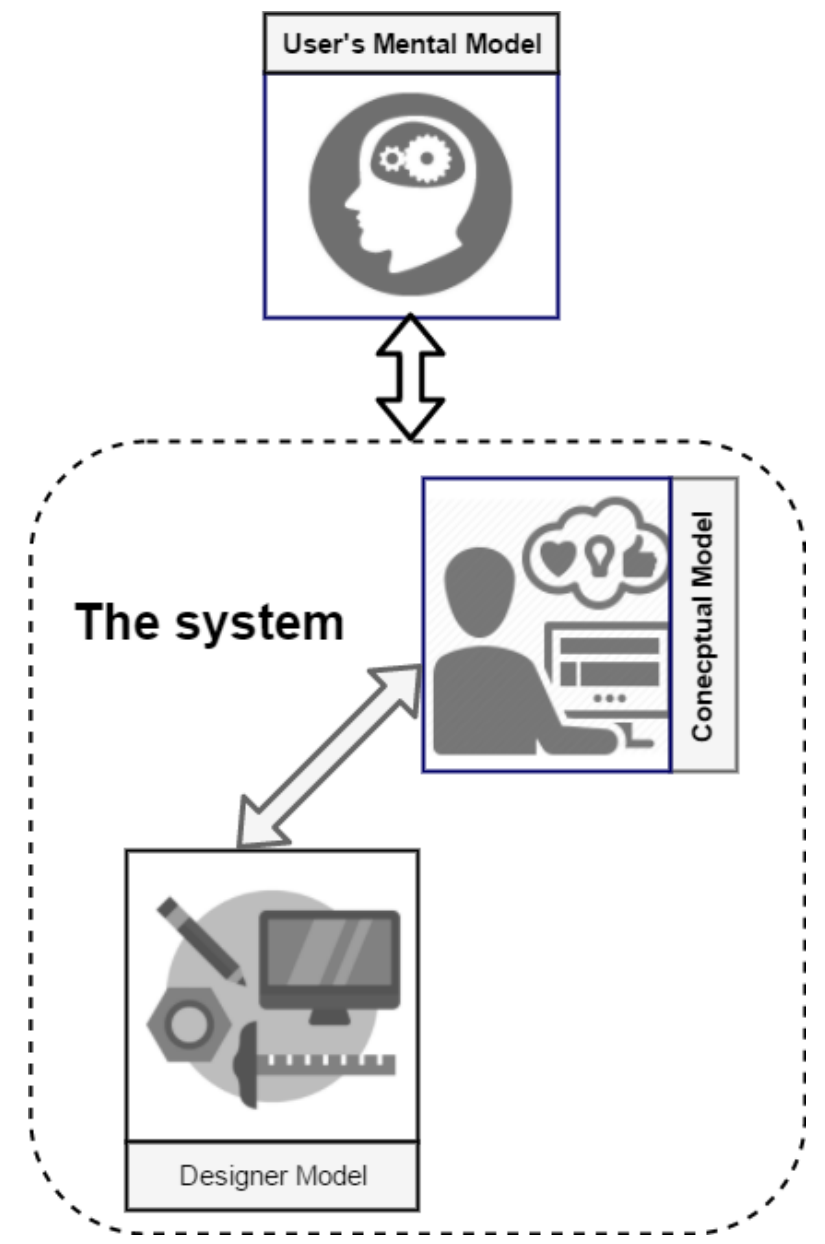

Figure 1. Relation between different models

The goal of the proposed methodology is to impact on the user's mental model to perceive the conceptual model represented in the system. The methodology shown in Fig.2 indicates the steps to be taken when designing a UI.

The first step is to identify the end goal or what users need to achieve by interacting with the interface to be built. An image or icon of the physical object needs to be placed in the main control view, so users can quickly navigate to the section they need.
The second step is to find all variations that can be controlled to achieve the end goal. It will be useful to find physical objects in real life representing those variations

The third step is to rename variations found in the second step into terminologies end users understand; it is also useful, if possible, to find physical objects in real life representing those variations.

The fourth step is to add control elements to the interface, decorated with icons and labels based on the outcome of the previous two steps. The more control elements represent actions in real life, the better.

The fifth step is to provide a mechanism that shows the system status in the language end users understand.

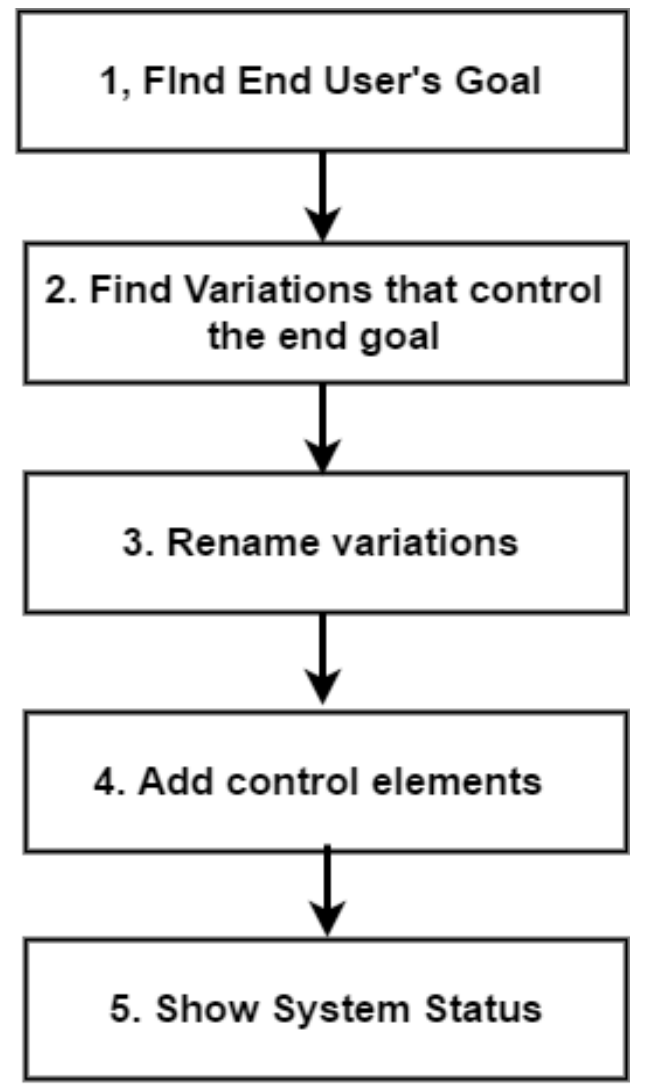

Figure 2. Steps of the proposed approach Example

In this section, we provide an example that shows how to apply the proposed approach in building an interface for controlling the position of front car seats.

The first step (Find the end goal): The end goal is to set the position of the seat to achieve the maximum level of comfort. Placing a car seat picture in the main control panel will be the best choice to get user attention. 
The second step: There are three different parts in the front car seats, which users control to obtain a position achieving the maximum level of comfort, as shown in fig.3.

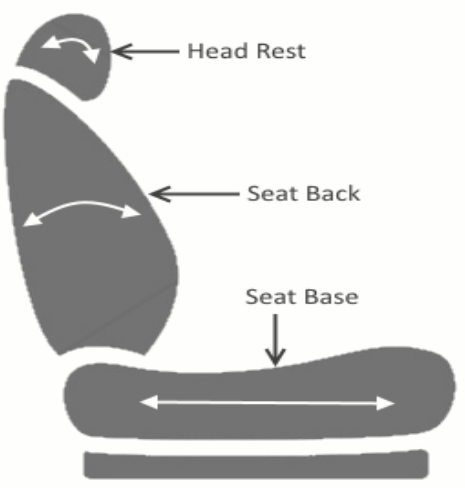

Figure 3. Steps of the proposed approach steps

The third step: There is no need to rename variations mentioned in the previous step, as end users are aware of those terms. It will be useful to associate an image of each variation with control elements in the next step.

The fourth step: Using sliders as control elements in the user interface for all variations is very similar to manual actions performed to adjust the seat position. Fig. 4 shows the control elements in the user interface.

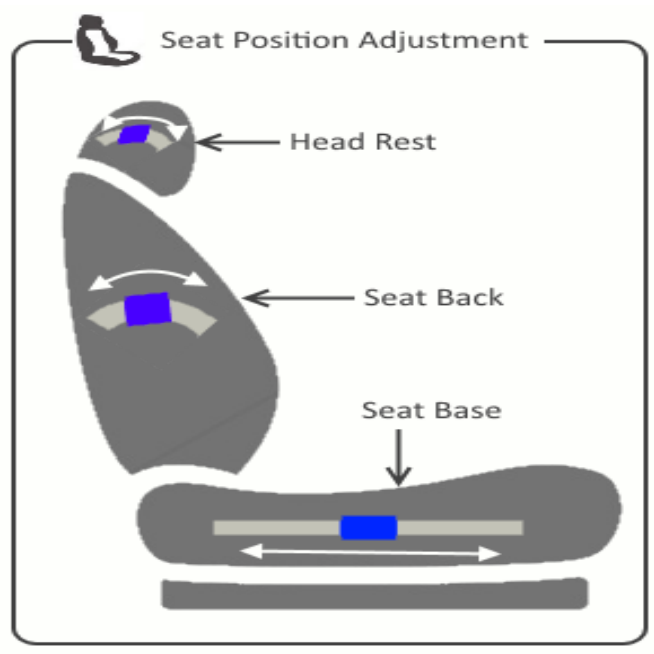

Figure 4. Placing control elements

The fifth step: Users need to be updated whether the system has succeeded in adjusting the seat position or not. This can be achieved by changing the sliding button background color to red, to indicate a failure in adjusting the position (Fig. 5). Alternatively, the background color will blink between green and grey for 2 seconds, to indicate that the system has succeeded in adjusting the seat to the set position.

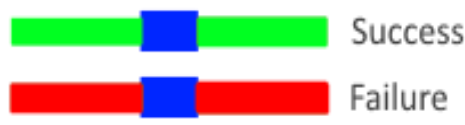

Figure 5. System Status Representation

\subsection{Evaluation}

It is essential to have end users evaluate the developed interface, in order to measure the effectiveness of the approach. The evaluation was carried out by developing a questionnaire to be answered by ten participating users. The questionnaire was constructed based on the ten usability heuristics for user interface design; all the questions were either closed questions, yes/no questions, or asking participants to give a rating between one and ten (where 10 refers to good and one refers to bad). Table 1 shows all of the questions and results.

Table 1. Evaluation Questionnaire

\begin{tabular}{|c|c|}
\hline Question & Result \\
\hline $\begin{array}{l}\text { How easy was it to find the interface for } \\
\text { seat position adjustment? } \\
\text { 1: Very difficult } \\
\text { 10: Very easy }\end{array}$ & Average: 10 \\
\hline $\begin{array}{l}\text { How do you describe the representation in } \\
\text { the interface for different parts of the front } \\
\text { car seat? } \\
\text { 1: No match } \\
\text { 10: Strongly Match }\end{array}$ & Average: 9.5 \\
\hline $\begin{array}{c}\text { How do you rate the simplicity of the } \\
\text { interface? } \\
\text { 1: Complex } \\
\text { 10: very simple }\end{array}$ & Average: 9.5 \\
\hline $\begin{array}{l}\text { How do you describe the flexibility of the } \\
\text { interface (e.g. it allows the position to be } \\
\text { easily restored to the previous state)? } \\
\text { 1: Not flexible } \\
\text { 10: Flexible }\end{array}$ & Average: 8 \\
\hline $\begin{array}{l}\text { Did you have to remember all the steps } \\
\text { required to use the interface at the second } \\
\text { attempt? }\end{array}$ & $\begin{array}{c}\text { Yes (Count): } 10 \\
\text { No (Count): } 0\end{array}$ \\
\hline $\begin{array}{l}\text { Did you notice that the system alerts you in } \\
\text { case of a failure? }\end{array}$ & $\begin{array}{l}\text { Yes (Count): } 9 \\
\text { No (Count): } 1\end{array}$ \\
\hline $\begin{array}{c}\text { Did you find any irrelevant information in } \\
\text { the interface? }\end{array}$ & $\begin{array}{l}\text { Yes (Count): } 10 \\
\text { No (Count): } 0\end{array}$ \\
\hline
\end{tabular}

\section{Related Work}

Dingli and Cassar [7] proposed an intelligent framework for website usability. The results have shown that the framework models human judgement when detecting usability violations.

Rule, Tabard, and Hollan [8] conducted an exploratory study demonstrating that users provided with a visual history 
containing small images can reconstruct detailed mental contexts of computer work.

O'Brien, Rogers, and Fisk [9] proposed a framework for intuitive human-computer interaction with specific attributes to allow designers to create products that elicit the targeted experience. Core concepts supporting this framework include finding user goals, carrying out well-learned behavior, determining what to do next, metacognition, knowledge in the head, and knowledge of the world.

\section{Conclusion and Future Work}

This paper has provided an approach that facilitates mental model mapping in a user interface. The approach was applied in building an interface for controlling positions of front car seats. The interface was evaluated using a questionnaire based on the ten usability heuristics, as there are strong links between them and mind mapping. Results have shown that these ten heuristics are met when applying the proposed approach. Future work will involve applying the approach to different domains to ensure that the approach is valid in all domains.

\section{References}

[1] Immersion (2012) Mental Model White Paper. February 2012.

[2] A. Kurtz (2017) Mental Models - A Theory Critique. The Open University.

[3] Mental Models and Reasoning (2006) What are the mental model. [Online] Available from: http://mentalmodels.princeton.edu/about/ [Accessed: 5th Jan 2017]

[4] Johnson-Laird (1983). Mental Model. Cambridge University Press.

[5] A. Khella (2002) Knowledge and Mental Models in HCI. The Human-Computer Interaction Lab at University of Maryland. [Online] Available from: https://www.cs.umd.edu/class/fall2002/cmsc838s/tichi/intro. html [Accessed: 20th Dec 2016]

[6] J. Nielsen (1995) 10 Usability Heuristics for User Interface Design. Nielsen Norman Group. [Online] Available from: https://www.nngroup.com/articles/ten-usabilityheuristics/ [Accessed: 5th Jan 2017]

[7] A. Dingli, and S. Cassar (2014) An Intelligent Framework for Website Usability. Advances in Human Computer Interaction, vol. 2014, Article ID 479286

[8] A. Rule, A. Tabard, and J. Hollan (2017) Using Visual Histories to Reconstruct the Mental Context of Suspended Activities, Human-Computer Interaction Journal
[9] M. O’Brien, A. Rogers, W. Fisk (2008). Developing a Framework for Intuitive Human-Computer Interaction. Proceedings of the Human Factors and Ergonomics Society Annual Meeting. Human Factors and Ergonomics Society. Annual Meeting, 52(20), 1645-1649. 